\title{
Vacuum Instability and Pair Nucleation in a Dissipative Medium
}

\author{
Roberto Iengo \\ International School for Advanced Studies, Via Beirut 4, 34014 Trieste (Italy) \\ and INFN - Sezione di Trieste, 34100 Trieste (Italy) \\ Giancarlo Jug日 \\ Max-Planck Institut für Physik Komplexer Systeme, Außenstelle Stuttgart \\ Heisenbergsrt. 1, Postfach 800665, D-70569 Stuttgart (Germany)
}

(September 1, 2018)

\begin{abstract}
We present a systematic and unifying treatment of the problem of spontaneous nucleation of particle-antiparticle pairs in a $(2+1)$-dimensional system due to a static and uniform electromagnetic-like field, in the presence of quantum dissipation. We first describe a direct derivation of the Caldeira-Leggett type of mechanism for quantum dissipation within the context of string theory and of the ensuing Born-Infeld action, pointing out the difference with the physical context in which vacuum decay can occur. We then evaluate the particle-antiparticle pair production rate, working out all the details of the calculation and including also the effects of a possible periodic background potential and of the Coulomb-like particle-antiparticle attraction. The former induces a dissipation-driven localization which interferes with the effect of the driving electric-like field. We also hint at a possible application to the problem of the decay of a supercurrent in a superconducting thin film due to vortex-antivortex nucleation in the presence of a pinning lattice.
\end{abstract}

PACS numbers: 11.10.-z, 11.25.-w, 05.30.-d, 74.60.Ge

Keywords: constant-field pair-creation, quantum dissipation

\section{INTRODUCTION}

Dissipative systems are ubiquitous in nature [罒]; of particular relevance in theoretical physics is "quantum dissipation", a relatively novel field of research [2] stemming from the seminal work of Caldeira and Leggett (CL) [3] and which is of course of great relevance for low-temperature condensed-matter physics. Surprisingly, quantum dissipation also appears to be built-in in the dynamics of string theory [1.,5].

In this paper we systematically study the role of quantum dissipation on the instability of the "vacuum", driven by a background uniform electromagnetic-like field. This issue is 
strictly related to the evaluation of the (one-loop) effective action of a constant electromagnetic field, that is the effective action induced by the virtual particle-antiparticle pairs. In fact the vacuum-instability is due in general to matter-field pair-nucleation and corresponds, technically, to the imaginary part of the effective action. In particular, in the context of string theory [6], there has been a considerable amount of work in order to determine the effective action due to a background electromagnetic field [4.]7. Here we discuss and compare the role of quantum dissipation both in a context of string theory and in a context of a quantum field theory for typical condensed matter problems, pointing out the relevant differences and similarities.

Although parts of this paper do not contain, strictly-speaking, new results - since they can be found in the above-quoted string-theory papers on the one side and in a rather condensed way in recent publications by the present authors [8 [10] on the other side - we do think it is important to offer a systematic and unified treatment of quantum dissipation and vacuum decay which can be of interest to both the string-theory and the condensedmatter physics communities. Furthermore, we do believe that the blend of many ingredients as presented in this paper is novel, timely and compelling.

The organization of this article is indeed as follows. We first (Section II) discuss quantum dissipation in the context of string theory, and exhibit a direct computation which shows that the string degrees of freedom provide an ideal CL dissipative bath for the string's end-point. In Section III, we complete the discussion pertinent to string theory by showing how quantum dissipation in a totally relativistic context yields the so-called Born-Infeld effective action [11] for the electromagnetic field. Namely, in this context, dissipation affects the motion in the time- and in the space-coordinates on the same footing. In this case we find that there is no vacuum decay. However, in a field-theory context more specifically oriented towards condensed-matter situations, where quantum dissipation is originated by the interaction with a medium which has only space reality, the vacuum can indeed decay and the calculation for the associated rate of pair production is described in Section IV. There, we specialize the calculation for the case of two space dimensions, by recalling the relevance of this calculation for the vortex-nucleation in a thin superconducting film. In Section $\mathrm{V}$ we introduce a physics problem where quantum dissipation plays an essential role, namely the dissipation-driven localization transition in the presence of a periodic background potential. Finally, in Section VI, we describe the vacuum instability when both background uniform electromagnetic-like field and a periodic background potential are present. We also include the effect of a Coulomb-like attraction between the particle and antiparticle in a pair. In Section VII we present our conclusions, commenting on the applicability of our work to the problem of vortex-antivortex nucleation-induced decay of a supercurrent in a superconducting thin film where a periodic pinning lattice has been artificially introduced.

We point out from the outset that since the phenomenon of spontaneous pair-nucleation driven by a uniform and static field is a long-standing prediction of quantum field theory which has never been directly observed so far - to our knowledge - our work on quantum vortex-nucleation could represent the possibility for an experimental observation of this phenomenon. Since this observation would occur in a solid-state device, the role of quantum dissipation is compelling. 


\section{QUANTUM DISSIPATION IN STRING THEORY}

The results reported in this Section and in the following one can also be found in the papers by Fradkin and Tseytlin [4], by Abouelsaood, Callan, Nappi and Yost [7], and by Callan and Thorlacius [5]. However here we present an alternative derivation, making use of a direct and straightforward computation which has also the advantage of being easily compared with the standard Caldeira and Leggett formulation [3] and with the other physical contexts to be discussed in the rest of this paper. We begin by writing, quite generally, the (Euclidean) action for an open string

$$
\mathcal{S}_{E}=\int_{0}^{\tau} d t \int_{0}^{\pi} d \sigma\left(\frac{\rho}{2}\left(\frac{\partial X}{\partial t}\right)^{2}+\frac{\nu}{2}\left(\frac{\partial X}{\partial \sigma}\right)^{2}\right) .
$$

Here, $X=X_{\mu}(t, \sigma)$ are the space-time coordinates of the string, whereas $t$ plays the role of the Schwinger (Euclidean) proper time parameter and $\sigma$ parametrizes the extension of the string. We consider periodic boundary conditions in $t$, and Neumann boundary conditions at the end points in $\sigma:\left.\partial_{\sigma} X\right|_{\sigma=0}=\left.\partial_{\sigma} X\right|_{\sigma=\pi}=0$. The manifold spanned by the string during its motion in $t$ is thus an annulus. We have introduced two arbitrary parameters for generality's sake, $\rho$ playing the role of the mass density and $\nu$ that of an elastic constant. In the standard string theory [6], they are related to each other and to the string tension.

We now derive the dynamics of the end point of this string, say at $\sigma=0$, denoting $q(t) \equiv X(t, \sigma=0)$, by deriving the end point's effective action, $S(q)$. We begin by Fourier analysing in $\sigma$

$$
X(t, \sigma)=\sum_{n=0}^{\infty} X_{n}(t) \cos (n \sigma)
$$

and further we Fourier analyse in $t: X_{n}(t)=\sum_{k=-\infty}^{\infty} X_{n k} e^{i \frac{2 \pi k}{\tau} t}$. The Euclidean action is now

$$
\mathcal{S}_{E}=\frac{\pi}{4} \tau \nu \sum_{n=0} n^{2} X_{n 0}^{2}+\frac{\pi}{2} \tau \sum_{n=0} \sum_{k=1}\left(\rho\left(\frac{2 \pi k}{\tau}\right)^{2}+n^{2} \nu\right)\left|X_{n k}\right|^{2}
$$

and the effective end-point action is obtained by tracing out all string degrees of freedom, except those for the end point. We thus compute the constrained functional integral

$$
e^{-S(q)}=\mathcal{N} \int \prod_{n=0}\left\{d X_{n 0} \prod_{k=1} d^{2} X_{n k}\right\} e^{-\mathcal{S}_{E}} \delta\left(\sum_{n=0} X_{n 0}-q_{0}\right) \prod_{k=1} \delta\left(\sum_{n=0} X_{n k}-q_{k}\right),
$$

having introduced $q(t)=\sum_{k=-\infty}^{+\infty} q_{k} e^{i \frac{2 \pi k}{\tau} t}$. Now for each $k$ we evaluate the constrained functional integral, by replacing $X_{0 k}=q_{k}-\sum_{n=1} X_{n k}$. We have

$$
e^{-S\left(q_{k}\right)}=e^{-\frac{\pi}{2} \rho \tau\left(\frac{2 \pi k}{\tau}\right)^{2}\left|q_{k}\right|^{2}} \mathcal{N} \int \prod_{n=1} d^{2} X_{n k} \exp \left\{-X_{n k}^{*} B_{n l} X_{l k}-X_{n k}^{*} V_{n}-V_{n}^{*} X_{n k}\right\},
$$

where we have introduced the matrix $(n, l=1,2, \ldots, \infty)$ 


$$
B_{n l}=a(n) \delta_{n l}+a(0)
$$

and the vector $V_{n}=a(0) q_{k}$, which is actually independent of $n$. Here

$$
a(n)=\frac{\pi}{2} \tau\left(\rho\left(\frac{2 \pi k}{\tau}\right)^{2}+n^{2} \nu\right) .
$$

Completing the square, we find

$$
e^{-S\left(q_{k}\right)}=e^{-\frac{\pi}{2} \rho \tau\left(\frac{2 \pi k}{\tau}\right)^{2}\left|q_{k}\right|^{2}} \exp \left\{V_{n}^{*} B_{n l}^{-1} V_{l}\right\} .
$$

One can check that the inverse matrix is

$$
B_{n l}^{-1}=\frac{1}{a(n)} \delta_{n l}-\frac{a(0)}{a(n) Q a(l)},
$$

where we have set $Q=\sum_{n=0} \frac{a(0)}{a(n)}$. We get, therefore

$$
S\left(q_{k}\right)=\frac{1}{\sum_{n=0} 1 / a(n)}\left|q_{k}\right|^{2} .
$$

Going now to the continuum limit in $\sigma$, which corresponds to the case where the inner circular border of the annulus shrinks to zero and mathematically to the limit $\nu \rightarrow 0$ (but keeping $\rho \nu$ finite), we replace the sum over $n$ with an integral, to get (regardless of the sign of $k$ )

$$
S\left(q_{k}\right)=\frac{\pi \nu \tau / 2}{\int_{0}^{\infty} d x /\left(\frac{\rho}{\nu}\left(\frac{2 \pi k}{\tau}\right)^{2}+x^{2}\right)}\left|q_{k}\right|^{2}=\eta 2 \pi|k|\left|q_{k}\right|^{2} .
$$

Here $\eta=\sqrt{\rho \nu}$ is to be identified with the friction coefficient of the CL formulation of quantum dissipation [3]. Interestingly, we obtain pure dissipative dynamics for the endpoint. By Fourier transformation of this expression we get the standard form for the periodic case

$$
S(q)=\frac{\eta \pi}{4 \tau^{2}} \int_{0}^{\tau} d t \int_{0}^{\tau} d t^{\prime}\left(\frac{q(t)-q\left(t^{\prime}\right)}{\sin \left[\pi\left(t-t^{\prime}\right) / \tau\right]}\right)^{2} .
$$

Notice that the true CL dissipative action [3] is obtained only when the string coordinates are strictly space-like.

\section{EFFECTIVE ELECTROMAGNETIC ACTION IN STRING THEORY}

Since $X(t, \sigma)$, and thus also $q(t)$, represent the complete set of space-time coordinates, the effective action for the end point found in the previous Section II contains quantum dissipation also in the time coordinate, $q_{\text {time }}(t)$. In this situation it is immediate to work out the effective action pertinent to the situation where a uniform electromagnetic field is 
coupled only to the string's end point. Namely, let us write the full action for $q(t)$, including this coupling

$$
\begin{aligned}
S\left(q, A_{\mu}\right) & =\sum_{k=1}^{\infty}\left[\eta 2 \pi k\left|q_{\mu k}\right|^{2}+i F_{\mu \nu}(i 2 \pi k) q_{\mu k}^{*} q_{\nu k}\right] \\
& =\sum_{k=1}^{\infty} 2 \pi k q_{\mu k}^{*}\left\{\eta \delta_{\mu \nu}-F_{\mu \nu}\right\} q_{\nu k} .
\end{aligned}
$$

Therefore, we get the effective action $S_{\text {eff }}\left(A_{\mu}\right)$ (remembering that the result of the functional integration over the string coordinates $X$ is interpreted in string theory as the one-loop contribution to the effective action $S_{\text {eff }}$ ):

$$
S_{e f f}\left(A_{\mu}\right)=\mathcal{N} \int \mathcal{D} q e^{-S\left(q, A_{\mu}\right)}=\prod_{k=1}^{\infty} \operatorname{det}\left(\delta_{\mu \nu}-\frac{1}{\eta} F_{\mu \nu}\right)^{-1}=\sqrt{\operatorname{det}\left(\delta_{\mu \nu}-\frac{1}{\eta} F_{\mu \nu}\right)}
$$

We have used here the standard $\zeta$-function regularization. We have thus obtained the BornInfeld action [11] in a rather straightforward way. We notice that this action is real (at least for fields such that, generically speaking, $F<\eta$ ) and there is thus no vacuum decay.

\section{VACUUM INSTABILITY IN A DISSIPATIVE MEDIUM}

In the following, we discuss situations where dissipation does lead to a vacuum decay, that is when it appears only for the space coordinates. This is indeed the physical situation for a particle which during its space-time motion interacts with the harmonic oscillators of a suitable CL bath. The particle will feel interactions which will depend on its space position and will affect its space trajectory. In these situations the action for the time-like component of the coordinates is of the usual form, namely $\int_{0}^{\tau} d t \frac{1}{4}\left(\partial_{t} q_{\text {time }}(t)\right)^{2}$.

We consider here the space-time coordinate $q(t)$ to describe closed loops in space-time as a function of the Schwinger (Euclidean) proper time $t$. These will correspond to the vacuum fluctuations due to virtual creation and annihilation of particle-antiparticle pairs. Vacuum instability will manifest itself when the virtual loops extend to infinity, giving rise to an imaginary part for the vacuum energy $W_{0}$. The latter, before the introduction of dissipation, is by definition ( $T$ being the total time interval)

$$
W_{0}=\frac{1}{T} \operatorname{Tr} \ln \left(-\frac{1}{\gamma} \mathbf{D}^{2}-D_{\text {time }}^{2}+\mathcal{E}_{0}^{2}\right) .
$$

This equation represents the standard contribution to the vacuum energy of a complex scalar field, whose quanta constitute the particles-antiparticles. $D$ is here the covariant derivative, containing the electromagnetic field $A_{\mu}, \mathcal{E}_{0}$ is the activation energy - that is, in the relativistic field theory, the rest mass $m$ times $c^{2}$, the square velocity of light - and $\gamma=1 / c^{2}=m / \mathcal{E}_{0}$. In turn, we write

$$
\operatorname{Tr} \ln \frac{-D_{E}^{2}+\mathcal{E}_{0}^{2}}{\Lambda^{2}}=-\lim _{\epsilon \rightarrow 0} \int_{\epsilon}^{\infty} \frac{d \tau}{\tau} \operatorname{Tr}\left\{e^{-\left(-D_{E}^{2}+\mathcal{E}_{0}^{2}\right) \tau}-e^{-\Lambda^{2} \tau}\right\}
$$


where we have defined $D_{E}^{2}=\frac{1}{\gamma} \mathbf{D}^{2}+D_{\text {time }}^{2}$. Finally, we use the path-integral representation (evaluating the imaginary part $\Gamma / 2$ of the vacuum energy)

$$
\frac{\Gamma}{2}=\frac{1}{T} \operatorname{Im} \int_{0}^{\infty} \frac{d \tau}{\tau} \mathcal{N} \int_{q(0)=q(\tau)} \mathcal{D} q(t) e^{-S_{E}\left(q, A_{\mu}\right)} .
$$

Quantum dissipation is now introduced by writing the corresponding Euclidean action, that is (restricting the form Eq. (2.12) to the sole spatial coordinates)

$$
\begin{aligned}
S_{E}\left(q, A_{\mu}\right) & =\int_{0}^{\tau} d t\left\{\frac{1}{4}\left(\partial_{t} q_{\text {time }}\right)^{2}+\frac{\gamma}{4}\left(\partial_{t} \mathbf{q}\right)^{2}+i \partial_{t} q_{\mu} A_{\mu}(q)+\mathcal{E}_{0}^{2}\right\} \\
& +\frac{\eta \pi}{4 \tau^{2}} \int_{0}^{\tau} d t \int_{0}^{\tau} d t^{\prime}\left(\frac{\mathbf{q}(t)-\mathbf{q}\left(t^{\prime}\right)}{\sin \left[\pi\left(t-t^{\prime}\right) / \tau\right]}\right)^{2},
\end{aligned}
$$

which in terms of the Fourier components of $q(t)$ reads

$$
S_{E}\left(q, A_{\mu}\right)=\sum_{k=1}^{\infty}\left\{\frac{1}{2} \frac{(2 \pi k)^{2}}{\tau}\left|q_{\text {time } k}\right|^{2}+\left[\frac{\gamma}{2} \frac{(2 \pi k)^{2}}{\tau}+\eta 2 \pi k\right]\left|\mathbf{q}_{k}\right|^{2}-F_{\mu \nu}(2 \pi k) q_{\mu k}^{*} q_{\nu k}\right\}+\mathcal{E}_{0}^{2} \tau .
$$

We are interested in the dissipation-dominated case, corresponding to $\gamma \rightarrow 0$. We make use of a $\gamma \neq 0$ as a regulator for the intermediate calculations.

¿From here on, we concentrate on the case of two space dimensions, having in mind the possible application to vortex-antivortex pair nucleation in superconducting thin films (Section VII). The electromagnetic field is taken to have the electric components $\mathbf{E}=(E, 0)$ in the two-dimensional plane, and the magnetic component $B$ orthogonal to the plane. As it is known, and as was shown in Ref. [8], the effect of the magnetic part $B$ effectively amounts to a renormalization of the friction coefficient: $\eta \rightarrow\left(B^{2}+\eta^{2}\right) / \eta$. Therefore, in what follows we drop $B$ and simply denote by $\eta$ the renormalized dissipation coefficient. Notice that the electric field appears as an imaginary quantity in the Euclidean formulation, that is $F_{a \text { time }}=(i E, 0)$ with $a=x, y$.

As a first step in the computation, we perform the integral over $q_{\text {time }}$. We get, by shifting the integration variable and performing the Gaussian integration:

$$
\begin{aligned}
& \mathcal{N} \int_{q(0)=q(\tau)} \mathcal{D} q(t) e^{-S_{E}\left(q, A_{\mu}\right)}= \\
& =\left(\frac{1}{4 \pi \tau}\right)^{1 / 2} e^{-\mathcal{E}_{0}^{2} \tau} \mathcal{N} \prod_{k=1}^{\infty} \int d^{2} q_{x k} e^{-\left(\eta 2 \pi k-2 \tau E^{2}\right)\left|q_{x k}\right|^{2}} \int d^{2} q_{y k} e^{-\eta 2 \pi k\left|q_{y k}\right|^{2}} .
\end{aligned}
$$

The factor $\left(\frac{1}{4 \pi \tau}\right)^{1 / 2}$ is here the standard free-particle result for the path-integral over $q_{\text {time }}$. Now we come to the essential point of our computation. The integration over $q_{x k}$ gives rise to poles in the variable $\tau$, which in turn - when inserted in the integration over $\tau$ with a suitable $i \epsilon$ prescription $\left(\operatorname{Im} \frac{1}{x-i \epsilon}=\pi \delta(x)\right)$ - give rise to an imaginary part for the vacuum energy, Eq. (4.1). The dominant pole, corresponding to the minimal value of $\tau$ and thus giving the leading term in an expansion made up of further exponentially-suppressed contributions, comes from integrating over $q_{x 1}$ : 


$$
\int d^{2} q_{x 1} e^{-\left(\eta 2 \pi-2 \tau E^{2}\right)\left|q_{x 1}\right|^{2}}=\pi /\left(\eta 2 \pi-2 \tau E^{2}\right) .
$$

Thus we keep only the dominant pole $\tau=\tau_{1}=\pi \eta / E^{2}$. We are left with the task of computing the "entropy" due to the integration over the remaining $q_{x k}, q_{y k}$. On the pole, the action for the modes $q_{x k>1}$ is the same as for $E^{2}=0$, as it is for $q_{y k}$, but with $k \rightarrow k-1$. Thus we compute (for a one-dimensional case)

$$
Z_{0} \equiv \mathcal{N} \prod_{k=1} \int d^{2} q_{k} e^{-\eta 2 \pi k\left|q_{k}\right|^{2}}=\mathcal{N} \prod_{k=1} \frac{1}{2 \eta k}
$$

Bearing in mind the normalization for the free particle, $\mathcal{N} \prod_{k=1} \frac{\tau}{2 \pi \gamma k^{2}}=\left(\frac{\gamma}{4 \pi \tau}\right)^{1 / 2}$, we regularize the above expression by introducing a frequency cutoff $\Omega$ for the dissipation as well as reintroducing the inertial mass represented by $\gamma$. Thus we write

$$
\mathcal{N} \prod_{k=1} \frac{1}{2 \eta k} \rightarrow\left(\frac{\gamma}{4 \pi \tau}\right)^{1 / 2} \prod_{k=1}^{n^{*}}\left(1+\frac{\tau \eta}{\gamma \pi k}\right)^{-1}
$$

where $n^{*}=\Omega \tau_{1} / 2 \pi$ is a large integer. Therefore, by using the formula

$$
\prod_{k=1}^{n^{*}}\left(1+\frac{z}{k}\right)^{-1} \rightarrow\left(n^{*}\right)^{-z} \Gamma(z+1),
$$

and the large-argument expression for the $\Gamma$-function, we find

$$
Z_{0}(x) Z_{0}(y)=\frac{\eta}{2 \pi} e^{-\tau_{1} \Delta \mathcal{E}^{2}}
$$

Thus we see that the regularization cutoffs $\Omega$ and $\gamma$ are reabsorbed into a renormalization of the activation energy:

$$
\Delta \mathcal{E}^{2}=\frac{2 \eta}{\pi \gamma}\left(1+\ln \frac{\Omega \gamma}{2 \eta}\right) .
$$

Here, we will include $\Delta \mathcal{E}^{2}$ into a redefinition of the activation energy, $\mathcal{E}_{0}^{2} \rightarrow \mathcal{E}_{R}^{2}=\mathcal{E}_{0}^{2}+\Delta \mathcal{E}^{2}$, so that, effectively, $Z_{0}(x) Z_{0}(y)=\eta / 2 \pi$. Actually, an extra factor of $2 \eta$ is retrieved from the fact that in the zero-field case (used to fix the normalization) the $q_{x 1}$-mode contribution is assumed to be included. Since here we instead compute its contribution separately, we have to divide by $\int d^{2} q_{x 1} e^{-2 \pi \eta\left|q_{x 1}\right|^{2}}=1 / 2 \eta$.

Finally, by carrying out the imaginary part of the $\tau$-integral in Eq. (4.3), and factoring out the integration over the zero modes $q_{0}$, giving the space-time volume, we get the pairproduction rate per unit area

$$
\frac{\Gamma}{2 L^{2}}=\frac{E \sqrt{\eta}}{2 \pi} e^{-\frac{\pi \eta \mathcal{E}_{R}^{2}}{E^{2}}}
$$

This formula applies to vortex-nucleation in a superconducting thin film ( [8], Section VII), when we set $E=2 \pi J, J$ being the supercurrent number density and $\mathcal{E}_{R}$ a suitably renormalized nucleation energy. The remainder of the paper deals with the interesting problem of vacuum decay in a dissipative medium when a background periodic potential is also present. 


\section{DISSIPATION-DRIVEN LOCALIZATION TRANSITION}

This Section summarises, for the reader's convenience, some known facts about the problem of the diffusion of a quantum particle in a dissipative medium, in the presence of a periodic potential. In the next Section, we will adapt this discussion for the case of pair-nucleation in the presence of a background periodic potential, of the form

$$
V(\mathbf{q})=A_{0} \sum_{a}\left(1-\cos k q_{a}\right)
$$

where the sum extends over the space coordinates. As is well-known [12, 13], the motion of a quantum particle in a periodic potential in the presence of friction displays a localization transition whereby for $\eta<\eta_{c}$ the particle is mobile whilst for $\eta>\eta_{c}$ it is confined, $\eta_{c}=$ $k^{2} / 2 \pi=2 \pi / d^{2}$ being a sharp threshold independent of the potential's amplitude $A_{0}$ (here, $d=2 \pi / k$ is the potential's lattice parameter; we assume a square-lattice structure). This is reproduced by our formalism, with $\mathbf{E}=B=0$ and $\gamma \rightarrow 0$ in Eq. (4.5), with the confined phase treated within the variational "blocked-renormalization" scheme first introduced by Fisher and Zwerger [14, through which a "mass" is dynamically generated for the damped modes' propagator. We recall that the presence of the potential $V(\mathbf{q})$, Eq. (5.1), modifies the action by a term $\int_{0}^{\tau} d t V(\mathbf{q})$. In the next Section we will come back to the case $\mathbf{E} \neq 0$ to see how the presence of the periodic potential affects the pair production (the case $\mathbf{E}=0$ and $B \neq 0$ has been discussed in Ref. [15]).

It is actually useful to recall here some of the results of this Kosterlitz-like renormalization group (RG) analysis, as it will be of some use in our treatment for the nucleation rate (Section VI). In our notation, the motion of a quantum particle moving in a dissipative environment and subjected to a periodic potential (5.1) is described by the Euclidean action

$$
\mathcal{H}(\mathbf{q})=\sum_{n=1} \eta \tau \omega_{n} q_{a}\left(\omega_{n}\right) q_{a}^{*}\left(\omega_{n}\right)+\int_{0}^{\tau} d t A_{0} \sum_{a}\left(1-\cos k q_{a}\right)
$$

where $\omega_{n}=\frac{2 \pi}{\tau} n$, and $q\left(\omega_{n}\right)$ are the Fourier modes $q_{n}$. In the continuum limit, the bare propagator is

$$
\left\langle q_{a}(\omega) q_{a^{\prime}}^{*}\left(\omega^{\prime}\right)\right\rangle_{0}=\delta\left(\omega-\omega^{\prime}\right) \delta_{a a^{\prime}} \frac{1}{\eta|\omega|} f\left(\frac{|\omega|}{\Omega}\right),
$$

with $f(x)$ a cutoff function and $\Omega$ the same CL cutoff frequency as in Section IV. We can, for example, make use of a frequency-space RG procedure at this point, much as in the theory of the surface roughening transition [16]. Writing $q_{a}(t)=\hat{q}_{a}(t)+\delta q_{a}(t)$, where $\delta q_{a}(t)$ contains the modes with frequencies $\frac{\Omega}{b}<\omega<\Omega(b=1+\epsilon$, with $\epsilon \rightarrow 0)$, and integrating

out these modes one finds that the first-order contribution represents a renormalization of the amplitude $A_{0}$ in the action. For this we get:

$$
\hat{A}_{0}=A_{0} \exp \left\{-\frac{1}{2} k^{2}\left\langle\left(\delta q_{a}\right)^{2}\right\rangle_{0}\right\}=A_{0} e^{-\frac{1}{2 \pi \eta} k^{2} \ln (1+\epsilon)} .
$$

There is no renormalization of the friction coefficient, and this can be argued [14] to hold to all orders. The $\mathrm{RG}$ recursion relations are then, in differential form (with $d \ell=\ln (1+\epsilon)$ ): 


$$
\begin{aligned}
\frac{d A_{0}}{d \ell} & =-\frac{k^{2}}{2 \pi \eta} A_{0}+\cdots=-\frac{\eta_{c}}{\eta} A_{0}+\cdots \\
\frac{d \eta}{d \ell} & =0
\end{aligned}
$$

To solve these is straightforward in the mobile phase, $\eta<\eta_{c}=k^{2} / 2 \pi$ :

$$
A_{0 R}(\ell)=A_{0}(0) \exp \left\{-\frac{\eta_{c}}{\eta} \ell\right\}, \quad \eta(\ell)=\eta(0)
$$

when the dimensionless amplitude of the renormalized pinning potential, $\mathcal{A}_{0 R}(\ell)=$ $A_{0 R}(\ell) / \Omega(\ell)$, vanishes exponentially for increasing time-scales (here $\Omega(\ell)=\Omega e^{-\ell}$ ). However, in the confined phase, $\eta>\eta_{c}$, the amplitude diverges, the perturbation expansion in $A_{0}$ breaks down and the RG iteration must be interrupted. Since the non-renormalization of $\eta$ allows for no natural characteristic time-scale (unlike in a genuine Kosterlitz-Thouless transition) to be introduced, a dynamically-generated mass term is included in the free Hamiltonian [14]. Carrying out the RG procedure with a modified propagator

$$
\left\langle q_{a}(\omega) q_{a^{\prime}}^{*}\left(\omega^{\prime}\right)\right\rangle_{0}=\delta\left(\omega-\omega^{\prime}\right) \delta_{a a^{\prime}} \frac{1}{\eta|\omega|+M^{2}} f\left(\frac{|\omega|}{\Omega}\right),
$$

one arrives at the differential RG equation for $A_{0}$ :

$$
\frac{d A_{0}}{d \ell}=-\frac{\eta_{c}}{\eta} \frac{1}{1+\mu e^{\ell}} A_{0}+\cdots
$$

where $\mu$ is the dimensionless square "mass" $\mu=M^{2} / \eta \Omega$, which has the solution (assuming $\mu$ scale-independent):

$$
A_{0 R}(\ell)=A_{0} \exp \left\{-\frac{\eta_{c}}{\eta} \ln \left(\frac{1+\mu}{e^{-\ell}+\mu}\right)\right\},
$$

corresponding to the change of pinning potential amplitude following an integration of the degrees of freedom in the shell $\Omega(\ell)<\omega<\Omega$. The mass is then determined by a selfconsistent procedure (Feynman's variational principle) [14 minimising the system's free energy with respect to $\mu$, a procedure leading to

$$
\mu=\frac{A_{0} k^{2}}{\eta \Omega}(\mu)^{\eta_{c} / \eta}
$$

This equation has the solution $\mu=0$ in the mobile phase $\left(\eta<\eta_{c}\right)$ and, in the more interesting confined phase $\left(\eta>\eta_{c}\right)$ :

$$
\mu=\left(\frac{2 \pi A_{0}}{\Omega} \frac{\eta_{c}}{\eta}\right)^{\eta /\left(\eta-\eta_{c}\right)}
$$

This concludes the necessary repilogue of the dissipation-driven localization transition. 


\section{PAIR-PRODUCTION RATE IN THE PRESENCE OF A PERIODIC POTENTIAL}

We now come to the evaluation of $\Gamma$, in the presence of the periodic potential of Eq. (5.1), which calls for the implementation of a RG-type approach. For reasons related to the physics of vortices in a superconductor, we are in fact interested in the case of strong dissipation (confined phase, $\eta>\eta_{c}$ ). In the relevant case and with the electric-field $\mathbf{E}$ in the $x$-direction, the motion of the quantum particle in the orthogonal $y$-direction is as described in the previous Section V and with the dynamically-generated "mass" treatment. Along the $x$-direction, we have seen that the mode $q_{1}$ is driven to infinity, while all other $n>1$ modes remain bounded. We can regard the modes with $n>1$ as describing fluctuations around the mean trajectory of the nucleating pair. Therefore, with the notation of the previous Section, we take $\hat{q}(t)=q_{0}+q_{1} e^{i \omega_{1} t}+q_{1}^{*} e^{-i \omega_{1} t}$ as the RG survivor-modes and $q_{R}(t)$ as the remainder, RG-integrated coordinate modes which infinitesimal part is the $\delta q(t)$ introduced before. In order to take into account the effect of the periodic potential in the $x$-direction we assign the dynamically-generated mass also to $q_{R}$. Now, however, we must take into account that the amplitude of the potential as "seen" by $q_{R}$ still depends on $\hat{q}$. Therefore, the variationally-determined mass will be in the end evaluated self-consistently.

The effect of the dynamically-generated mass on the computation will be encoded in the renormalization of $A_{0}$ and in the evaluation of the "entropy" factor $Z$ due to the $n>1$ modes in the $x$-direction $\left(q_{R}\right)$, and to all $n>0$ modes in the $y$-direction. Since the modes in $q_{R}$ are purely Gaussian, we can now evaluate (for each space dimension) the correcting factor $B^{*}$ due to the dynamically-generated mass $M: Z=Z_{0} B^{*}$, where $Z_{0}$ is given by Eq.s (4.8) and (4.11) and where

$$
B^{*}=\prod_{n=1}^{n^{*}}\left(1+\frac{\tau M^{2}}{2 \pi \eta n}\right)^{-1}
$$

Making use of arguments similar to those seen for Eq.s (4.9) and (4.11) we get (in the limit where the pole value $\tau_{1}$ is very large)

$$
B^{*}=\sqrt{\frac{M^{2} \tau_{1}}{\eta}} e^{-\tau_{1} \Delta \tilde{\mathcal{E}}^{2}}
$$

where $\Delta \tilde{\mathcal{E}}^{2}=\frac{M^{2}}{2 \pi \eta}\left(1+\ln \frac{\Omega \eta}{M^{2}}\right)$ is to be regarded as a further renormalization of the activation energy.

Having treated the effect of the higher modes in $q_{R}$, we now discuss the dramatic dynamical effects of the unbounded $\hat{q}(t)$. The dynamics of these surviving modes is described by the Euclidean action $\hat{S}_{E}$ :

$$
\hat{S}_{E}=\left(2 \pi \eta-2 \tau E^{2}\right)\left|q_{1}\right|^{2}+A_{0 R} \int_{0}^{\tau} d t(1-\cos k \hat{q}(t))+\tau\left(\mathcal{E}_{R}^{2}+K \ln \frac{\left|q_{1}\right|}{a}\right)
$$

In this formula we have also introduced, for completeness, the Coulomb interaction between the particle and the antiparticle of the pair, in two space dimensions, a being a suitable 
ultraviolet cutoff. We treat this Coulomb interaction approximately, keeping only the dependence on the average particle-antiparticle distance, $\left|q_{1}\right| . A_{0 R}$ is given by Eq. (5.9), with $\ell=\ell^{*}=\ln n^{*} \rightarrow \infty$. We find $\left(J_{0}(x)\right.$ and $I_{0}(x)$ being Bessel functions)

$$
\int_{0}^{\tau} \cos k \hat{q}(t)=\tau J_{0}\left(2 k\left|q_{1}\right|\right) \cos k q_{0}
$$

and, averaging over the overall position $q_{0}$

$$
\hat{S}_{E}=\left(2 \pi \eta-2 \tau E^{2}\right)\left|q_{1}\right|^{2}-\ln \left(I_{0}\left(\tau A_{0 R} J_{0}\left(2 k\left|q_{1}\right|\right)\right)\right)+\tau\left[\mathcal{E}_{R}^{2}+K \ln \frac{\left|q_{1}\right|}{a}+A_{0 R}\right] .
$$

The integration $\int d^{2} q_{1} e^{-\hat{S}_{E}}$ and the subsequent integration over $\tau$, as in Eq.s (4.3) and (4.7), can then be performed by means of a saddle-point method, making use of the asymptotic formula for large argument

$$
I_{0}(x \tau) \rightarrow \frac{1}{\sqrt{2 \pi|x| \tau}} e^{|x| \tau} .
$$

Equating to zero the variation of $\hat{S}_{E}$ with respect to $\tau$ gives implicitely the value of $\ell_{N} \equiv\left|q_{1}\right|$ at the saddle point

$$
\ell_{N}^{2}=\frac{1}{2 E^{2}}\left(\mathcal{E}_{R}^{2}+K \ln \frac{\ell_{N}}{a}+A_{0 R}-\left|A_{0 R} J_{0}\left(2 k \ell_{N}\right)\right|\right) .
$$

This equation can be solved recursively. At the leading order we find the same solution of Section $\mathrm{V}$, namely $\ell_{N}^{2}=\frac{1}{2 E^{2}}\left(\mathcal{E}_{R}^{\prime 2}+A_{0 R}^{\prime}\right)$, now having set $\mathcal{E}_{R}^{\prime 2}=\mathcal{E}_{R}^{2}+K \ln \left(\ell_{0 N} / a\right)$ and $A_{0 R}^{\prime}=A_{0 R}-\left|A_{0 R} J_{0}\left(2 k \ell_{0 N}\right)\right|$ with $\ell_{0 N}=\left(\mathcal{E}_{R}^{2}+A_{0 R}\right) / 2 E^{2}$.

Similarly, the leading saddle-point value for $\tau$ is the same as in Section IV, $\tau_{1}=\pi \eta / E^{2}$. Thus, at the leading order the $q_{1}$ and $\tau$ integrals can be done as in Section IV. Therefore, we get

$$
\frac{\Gamma}{2 L^{2}}=B_{x}^{*} B_{y}^{*} \frac{E \sqrt{\eta}}{2 \pi} I_{0}\left(\frac{\pi \eta A_{0 R}}{E^{2}} J_{0}\left(2 k \ell_{N}\right)\right) \exp \left\{-\frac{\pi \eta\left(\mathcal{E}_{R}^{2}+K \ln \left(\ell_{N} / a\right)+A_{0 R}\right)}{E^{2}}\right\} .
$$

It is important to notice that, strictly speaking, the dynamically-generated "masses" are different in the $x$ - and $y$-directions. Moreover, we have yet to determine the self-consistent value of the mass for the $x$-direction; the latter can be carried out by repeating the variational calculation reviewed in Section $\mathrm{V}$ and by substituting for $A_{0}: A_{0} \rightarrow A_{0}\left|J_{0}\left(2 k \ell_{N}\right)\right|$. This, of course, holds good only away from the zeroes of the $J_{0}(x)$-function where also the whole saddle-point evaluation above should be modified. Still, these zeroes denote minima of the production rate (the latter being our main physical prediction here) where the pairnucleation has a deep drop. This is the main physical effect, and thus details of the dynamics inside these minima are believed to be irrelevant.

Our final formula for the production rate, neglecting for simplicity's sake possible differences between the $x$ - and $y$-dynamically-generated masses, and using the dimensionless quantity $\mu=M^{2} / \Omega \eta$, is:

$$
\frac{\Gamma}{2 L^{2}}=\frac{\mu \Omega}{2 E} \eta^{3 / 2} I_{0}\left(\frac{\pi \eta A_{0 R}}{E^{2}} J_{0}\left(2 k \ell_{N}\right)\right) \exp \left\{-\frac{\pi \eta\left(\mathcal{E}_{R}^{2}+K \ln \left(\ell_{N} / a\right)+A_{0 R}\right)}{E^{2}}\right\} .
$$




\section{POSSIBLE APPLICATION TO VORTEX-NUCLEATION INDUCED DECAY OF A SUPERCURRENT IN A SUPERCONDUCTING THIN-FILM.}

In some recent papers [8,9], we have argued that our theory could find its most direct application (that is, beyond its intrinsic theoretical physics value) in a specific solid-state physics problem. Namely, we have considered the situation in which - in the absence of an external magnetic field - a supercurrent is maintained in a thin superconducting film at very low temperatures, where the vortex dynamics is intrinsically quantum. We envisage films made up of some high- $T_{c}$ material, in order to afford high critical currents. Experiments [17] have shown evidence for a residual low-temperature resistance which depends very non-linearly on the current, $I$. We have argued that, beside the standard edge-tunneling mechanism [18.19,10], spontaneous vortex-antivortex nucleation should also play an important role in inducing supercurrent decay (see also Ref. [20] for earlier work).

We very briefly recall how the problems discussed in the previous Sections can find their implementation in vortex physics [21]. Vortices in a superconducting material experience a Magnus force [21,22] (even in the absence of an external magnetic field) which is the exact analogue of the electromagnetic force for point-charges and couples to the vortices via their topological charge. One can show that while the standard expression for the magnetic-like Magnus field, orthogonal to the film's plane in our geometry, is given by $B_{M}=2 \pi s \rho_{s}^{(3)}$ ( $s$ being the film's thickness, $\rho_{s}^{(3)}$ the three-dimensional superfluid number density), the electric-like part is proportional and orthogonal to the applied supercurrent number density $\mathbf{J}$, that is $\mathbf{E}_{M}=\times 2 \pi \mathbf{J}$ [8]. The Magnus force is then of the Lorentz form, namely $\mathbf{F}_{M}=\mathbf{E}_{M}-\dot{\mathbf{q}} \times \mathbf{B}_{M}$. Therefore, we could regard the vortex-antivortex pairs as point-like particle-antiparticle pairs coupling to the static e.m.-like Magnus field, $A_{M \mu}$. The spontaneous nucleation of these pairs in the bulk of the superconducting film is therefore a further cause of supercurrent decay and another important source for the film's residual resistance. The decay rate is given by Eq. (4.13) in our theory, in the absence of thermal fluctuations and of a pinning potential. We notice that suggestions for a vortex-antivortex nucleation mechanism for supercurrent decay in superconducting films were already put forth by Halperin and Nelson [23] in the context of a temperature-driven Kosterlitz-Thouless unbinding of vortex-antivortex pairs. In our theory, the components of each pair eventually decouple (even at zero temperature and for any $B_{M}$ ) due to quantum dissipation, which is the fundamental origin of the residual resistance [8]. The data of Ref. [17] can be fitted by a law of the type $R=R_{0} \exp \left\{-a /\left(I-I_{0}\right)^{b}\right\}$, with $a, b$ and $I_{0}$ independent fitting parameters. This seems to agree with our formulas.

A much-researched way of hindering the (thermal- or quantum-) motion of the vortices (thus reducing the resistance) is represented by the introduction of pinning centers [21]. In fact, periodic pinning would depress the vortices mobility. However, the main focus of the present paper is rather on the vortex-antivortex nucleation. According to our results of Section VI, the nucleation would be depressed for $A_{0}>0$ and favoured for $A_{0}<0$, the latter case being probably the more relevant one as it corresponds to the expectation that the presence of pinning centers could actually aid the nucleation. In any case, current-dependent oscillations linked to the periodicity of the pinning lattice would modulate the nucleation rate, see Eq. (6.9), and eventually could also appear in the induced resistance. ¿From our estimates [9] of the material parameters entering the phenomenological description of 
the quantum nucleation we have predicted that the oscillations ought to be observable. Superconducting films containing lateral superlattice arrays of void or normal-metal holes have in fact begun to be fabricated and studied [24].

In conclusion, we have presented in a new light and context a systematic study of particleantiparticle pair nucleation in the presence of static fields and of a dissipative medium. We have shown that pure CL quantum dissipation corresponds to the coupling to a space-like string, while full relativistic space-time-like strings give rise to no vacuum decay. We have applied our formalism to evaluate the pair-production rate induced by an e.m.-like field and demonstrated that in the presence of a background periodic potential oscillations connected to the underlying potential's periodicity will show up in the vacuum decay rate. Our theory could have interesting consequences for suitably-prepared superconducting thin films.

\section{ACKNOWLEDGEMENTS}

R.I. is grateful to the Max-Planck Institut für Physik Komplexer Systeme, Außenstelle Stuttgart, where this work was completed, for kind hospitality; G.J. is grateful to the International School for Advanced Studies (SISSA), Trieste, where part of this work was carried out, for hospitality. We are grateful to Dieter Weiss for pointing out Ref. 24] to us. We

acknowledge support from EC contracts No.s CHRX-CT920035, ERBFMRXCT96-0045 and ERB4001GT957255. 


\section{REFERENCES}

* On leave of absence from: Istituto di Scienze Matematiche, Fisiche e Chimiche, Università di Milano a Como, Via Lucini 3, 22100 Como (Italy) (permanent address).

[1] Aristotele, Physica VIII (384-322 b.C.).

[2] U. Weiss, Quantum Dissipative Systems (World Scientific, Singapore 1993).

[3] A.O. Caldeira and A.J. Leggett, Ann. Phys. 149, 374 (1983).

[4] E.S. Fradkin and A.A. Tseytlin, Phys. Lett. B 163, 123 (1985).

[5] C.G. Callan and L. Thorlacius, Nucl. Phys. B 329, 117 (1990).

[6] For useful reviews, see, e.g.: J.H. Schwarz, Physics Reports 89, 223 (1982); A.M. Polyakov, Gauge Fields and Strings (Harwood, Chur 1987); M.B. Green,J.H. Schwarz and E. Witten, Superstring Theory (Cambridge university Press, 1987).

[7] A.A. Abouelsaood, C.G. Callan, C.R. Nappi and S.A. Yost, Nucl. Phys. B 280599 (1987).

[8] R. Iengo and G. Jug, Phys. Rev. B 52, 7536 (1995).

[9] R. Iengo and G. Jug, Phys. Rev. B 54, 9465 (1996).

[10] R. Iengo and G. Jug, Phys. Rev. B 54, 13207 (1996).

[11] M. Born and L. Infeld, Proc. R. Soc. London A 144, 425 (1934).

[12] A. Schmid, Phys. Rev. Lett. 51, 1506 (1983).

[13] F. Guinea, V. Hakim and A. Muramatsu, Phys. Rev. Lett. 54, 263 (1985).

[14] M.P.A. Fisher and W. Zwerger, Phys. Rev. B 32, 6190 (1985).

[15] C.G. Callan, A.G. Felce and D.E. Freed, Nucl. Phys. B 392551 (1993).

[16] P. Nozières, in: Solids far from Equilibrium, G. Godrèche (Ed.) (Cambridge University Press, 1992).

[17] C. Paracchini and L. Romanò, Physica C 262, 207 (1996); C. Paracchini, L. Romanò, C.A. Borri and M. Salvato, Physica C 258, 222 (1996).

[18] P. Ao and D.J.Thouless, Phys. Rev. Lett. 70, 2158 (1993).

[19] M.J. Stephen, Phys. Rev. Lett. 72, 1534 (1994).

[20] P. Ao, J. Low Temp. Phys. 89, 543 (1992).

[21] For some recent surveys of this field, see: G. Blatter, M.V. Feigel'man, V.B. Geshkenbein, A.I. Larkin and V.M. Vinokur, Rev. Mod. Phys. 66, 1125 (1994); E.H. Brandt, Rep. Progr. Phys. 58, 1465 (1995).

[22] P. Ao and D.J. Thouless, Phys. Rev. Lett. 72, 132 (1994).

[23] B.I. Halperin and D.R. Nelson, J. Low Temp. Phys. 36, 599 (1979).

[24] V.V. Moshchalkov, M. Baert, V.V. Metlushko, E. Rosseel, M. van Bael, K. Temst, X.G. Qiu, R. Jonckheere and Y. Bruynsearede, Jpn. J. Appl. Phys. 34, 4559 (1995); and references therein. 